\title{
On a General Class of Multiple Eulerian Integrals with Multivariable I-Functions
}

By Frederic Ayant

Abstract- Recently, Raina and Srivastava and Srivastava and Hussain have provided closed-form expressions for a number of a Eulerian integral involving multivariable $\mathrm{H}$-functions. Motivated by these recent works, we aim at evaluating a general class of multiple Eulerian integrals concerning the product of two multivariable l-functions defined by Prathima et al. [6], a class of multivariable polynomials and the spheroidal function. These integrals will serve as a capital formula from which one can deduce numerous integrals.

Keywords: multivariable I-function, multiple eulerian integrals, class of polynomials, spheroidal functions, I-function of two variables, I-function of one variable.

GJSFR-F Classification: MSC 2010: 05C4

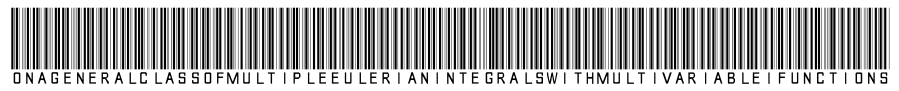

Strictly as per the compliance and regulations of:

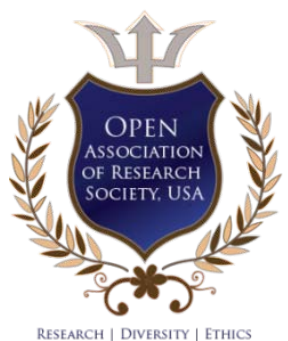

(C) 2018. Frederic Ayant. This is a research/review paper, distributed under the terms of the Creative Commons AttributionNoncommercial 3.0 Unported License http://creativecommons.org/licenses/by-nc/3.0/), permitting all non commercial use, distribution, and reproduction in any medium, provided the original work is properly cited. 

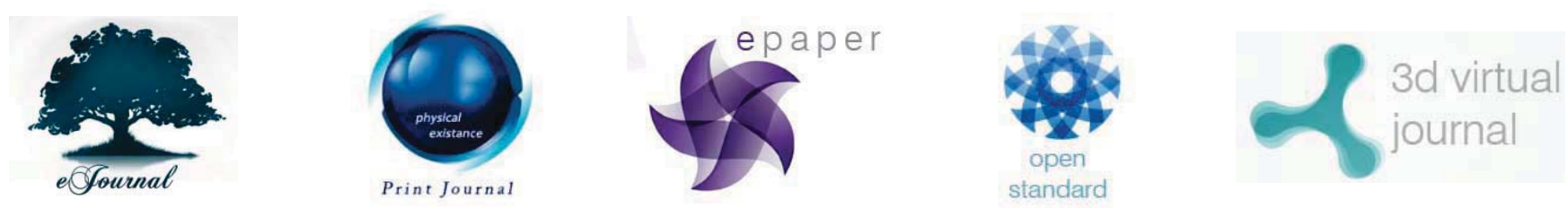

\title{
On a General Class of Multiple Eulerian
} Integrals with Multivariable I-Functions

\author{
Frederic Ayant
}

Abstract- Recently, Raina and Srivastava and Srivastava and Hussain have provided closed-form expressions for a number of a Eulerian integral involving multivariable $\mathrm{H}$-functions. Motivated by these recent works, we aim at evaluating a general class of multiple Eulerian integrals concerning the product of two multivariable l-functions defined by Prathima et al. [6], a class of multivariable polynomials and the spheroidal function. These integrals will serve as a capital formula from which one can deduce numerous integrals.

Keywords: multivariable l-function, multiple eulerian integrals, class of polynomials, spheroidal functions, Ifunction of two variables, l-function of one variable.

\section{Introduction And Prerequisites}

The well-known Eulerian Beta integral [5]

$$
\int_{a}^{b}(z-a)^{\alpha-1}(b-t)^{\beta-1} \mathrm{~d} t=(b-a)^{\alpha+\beta-1} B(\alpha, \beta)(\operatorname{Re}(\alpha)>0, \operatorname{Re}(\beta)>0, b>a)
$$

Is a basic result of evaluation of numerous other potentially useful integrals involving various special functions and polynomials. The mathematicians Raina and Srivastava [7], Saigo and Saxena [9], Srivastava and Hussain [14], Srivastava and Garg [13] et cetera have established a number of Eulerian integrals involving the various general class of polynomials, Meijer's G-function and Fox's H-function of one and more variables with general arguments. Recently, several Author study some multiple Eulerian integrals, see Bhargava [2], Goyal and Mathur [4] and others. The aim of this paper is to obtain general multiple Eulerian integrals of the product of two multivariable I-functions defined by Prathima et al [7], a general class of multivariable polynomials [12] and the spheroidal functions.

The spheroidal function $\psi_{\alpha n^{\prime}}(c, \eta)$ of general order $\alpha>-1$ can be expanded as ([10], [18]).

$$
\psi_{\alpha n^{\prime \prime}}(c, \eta)=\frac{i^{n^{\prime \prime}} \sqrt{2 \pi}}{V_{\alpha n^{\prime \prime}(c)}} \sum_{k=0, o r 1}^{\infty_{*}} a_{k}\left(c \mid \alpha n^{\prime \prime}\right)(c \eta)^{-\alpha-\frac{1}{2}} J_{k+\alpha+\frac{1}{2}}(c \eta)
$$

Which represents the expression uniformly on $(\infty, \infty)$, where the coefficients $a_{k}\left(c \mid \alpha n^{\prime \prime}\right)$ satisfy the recursion formula and the asterisk over the summation sign indicate that the sum is taken over only even or odd values of according as $n^{\prime \prime}$ is even or odd. As $c \rightarrow 0, a_{k}\left(c \mid \alpha n^{\prime \prime}\right) \rightarrow 0, k \neq n^{\prime \prime}$

The class of multivariable polynomials defined by Srivastava [12], is given in the following manner: 


$$
S_{N_{1}, \cdots, N_{v}}^{\mathfrak{M}_{1}, \cdots, \mathfrak{M}_{\mathfrak{v}}}\left[y_{1}, \cdots, y_{v}\right]=\sum_{K_{1}=0}^{\left[N_{1} / \mathfrak{M}_{1}\right]} \cdots \sum_{K_{v}=0}^{\left[N_{v} / \mathfrak{M}_{\mathfrak{v}}\right]} \frac{\left(-N_{1}\right)_{\mathfrak{M}_{1} K_{1}}}{K_{1} !} \cdots \frac{\left(-N_{v}\right)_{\mathfrak{M}_{\mathfrak{v}} K_{v}}}{K_{v} !} A\left[N_{1}, K_{1} ; \cdots ; N_{v}, K_{v}\right] y_{1}^{K_{1}} \cdots y_{v}^{K_{v}}
$$

where $\mathfrak{M}_{1}, \cdots, \mathfrak{M}_{\mathfrak{v}}$ are arbitrary positive integers and the coefficients $A\left[N_{1}, K_{1}\right.$; $\left.\cdots ; N_{v}, K_{v}\right]$ are arbitrary real or complex constants.

We shall note $a_{v}^{\prime}=\frac{\left(-N_{1}\right)_{\mathfrak{M}_{1} K_{1}}}{K_{1} !} \cdots \frac{\left(-N_{v}\right)_{\mathfrak{M}_{\mathfrak{v}} K_{v}}}{K_{v} !} A\left[N_{1}, K_{1} ; \cdots ; N_{v}, K_{v}\right]$

The I-function of several variables is a generalization of the multivariable $\mathrm{H}$ function studied by Srivastava et Panda [16,17]. The multiple Mellin-Barnes integrals occurring in this paper will be referred to as the multivariables $I$-function of $r$-variables throughout our present study and will refer and represented as follows:

$$
\begin{aligned}
& \bar{I}\left(z_{1}, \cdots, z_{r}\right)=\bar{I}_{p, q: m_{1}, q_{1} ; \cdots ; p_{r}, q_{r}}^{0, n: m_{1} ; \cdots ; m_{r}, n_{r}}\left(\begin{array}{c|c}
\mathrm{z}_{1} & \left(\mathrm{a}_{j} ; \alpha_{j}^{(1)}, \cdots, \alpha_{j}^{(r)} ; A_{j}\right)_{1, p}: \\
\cdot & \\
\cdot & \\
\cdot & \left(\mathrm{b}_{j} ; \beta_{j}^{(1)}, \cdots, \beta_{j}^{(r)} ; B_{j}\right)_{1, q}: \\
\mathrm{z}_{r} &
\end{array}\right. \\
& \left.\begin{array}{c}
\left(\mathrm{c}_{j}^{(1)}, \gamma_{j}^{(1)} ; C_{j}^{(1)}\right)_{1, n_{1}},\left(c_{j}^{(1)}, \gamma_{j}^{(1)} ; C_{j}^{(1)}\right)_{n_{1}+1, p_{1}} ; \cdots ;\left(c_{j}^{(r)}, \gamma_{j}^{(r)} ; C_{j}^{(r)}\right)_{1, n_{r}},\left(c_{j}^{(r)}, \gamma_{j}^{(r)} ; C_{j}^{(r)}\right)_{n_{r}+1, p_{r}} \\
\left(\mathrm{~d}_{j}^{(1)}, \delta_{j}^{(1)} ; 1\right)_{1, m_{1}},\left(d_{j}^{(1)}, \delta_{j}^{(1)} ; D_{1}\right)_{m_{1}+1, q_{1}} ; \cdots ;\left(d_{j}^{(r)}, \delta_{j}^{(r)} ; 1\right)_{1, m_{r}},\left(d_{j}^{(r)}, \delta_{j}^{(r)} ; D_{r}\right)_{m_{r}+1, q_{r}}
\end{array}\right) \\
& =\frac{1}{(2 \pi \omega)^{r}} \int_{L_{1}} \cdots \int_{L_{r}} \psi\left(s_{1}, \cdots, s_{r}\right) \prod_{k=1}^{r} \theta_{k}\left(s_{k}\right) z_{k}^{s_{k}} \mathrm{~d} s_{1} \cdots \mathrm{d} s_{r}
\end{aligned}
$$

Where $\psi\left(s_{1}, \cdots, s_{r}\right), \theta_{i}\left(s_{i}\right), i=1, \cdots, r$ are given by :

$$
\begin{aligned}
\psi\left(s_{1}, \cdots, s_{r}\right) & =\frac{\prod_{j=1}^{n} \Gamma^{A_{j}}\left(1-a_{j}+\sum_{i=1}^{r} \alpha_{j}^{(i)} s_{j}\right)}{\prod_{j=n+1}^{p} \Gamma^{A_{j}}\left(a_{j}-\sum_{i=1}^{r} \alpha_{j}^{(i)} s_{j}\right) \prod_{j=1}^{q} \Gamma^{B_{j}}\left(1-b j+\sum_{i=1}^{r} \beta_{j}^{(i)} s_{j}\right)} \\
\theta_{i}\left(s_{i}\right) & =\frac{\prod_{j=1}^{n_{i}} \Gamma^{C_{j}^{(i)}}\left(1-c_{j}^{(i)}+\gamma_{j}^{(i)} s_{i}\right) \prod_{j=1}^{m_{i}} \Gamma\left(d_{j}^{(i)}-\delta_{j}^{(i)} s_{i}\right)}{\prod_{j=n_{i}+1}^{p_{i}} \Gamma^{C_{j}^{(i)}}\left(c_{j}^{(i)}-\gamma_{j}^{(i)} s_{i}\right) \prod_{j=m_{i}+1}^{q_{i}} \Gamma^{D_{j}^{(i)}}\left(1-d_{j}^{(i)}-\delta_{j}^{(i)} s_{i}\right)}
\end{aligned}
$$

For more details, see Prathima et al. [6].

Following the result of Braaksma [3] the I-function of $\mathrm{r}$ variables is analytic if :

$$
U_{i}=\sum_{j=1}^{p} A_{j} \alpha_{j}^{(i)}-\sum_{j=1}^{q} B_{j} \beta_{j}^{(i)}+\sum_{j=1}^{p_{i}} C_{j}^{(i)} \gamma_{j}^{(i)}-\sum_{j=1}^{q_{i}} D_{j}^{(i)} \delta_{j}^{(i)} \leqslant 0 ; i=1, \cdots, r
$$

The integral (1.4) converges absolutely if

$\left|\arg \left(z_{k}\right)\right|<\frac{1}{2} \Delta_{k} \pi, k=1, \cdots, r$ where

$$
\Delta_{k}=-\sum_{j=n+1}^{p} A_{j} \alpha_{j}^{(k)}-\sum_{j=1}^{q} B_{j} \beta_{j}^{(k)}+\sum_{j=1}^{m_{k}} \delta_{j}^{(k)}-\sum_{j=m_{k}+1}^{q_{k}} D_{j}^{(k)} \delta_{j}^{(k)}+\sum_{j=1}^{n_{k}} C_{j}^{(k)} \gamma_{j}^{(k)}-\sum_{j=n_{k}+1}^{p_{k}} C_{j}^{(k)} \gamma_{j}^{(k)}>0
$$


and if all the poles of (1.6) are simple, then the integral (1.4) can be evaluated with the help of the residue theorem to give

$$
\bar{I}\left(z_{1}, \cdots, z_{r}\right)=\sum_{G_{k}=1}^{m_{k}} \sum_{g_{k}=0}^{\infty} \phi \frac{\prod_{k=1}^{r} \phi_{k} z_{k}^{\eta_{G_{k}, g_{k}}}(-)^{\sum_{k=1}^{r} g_{k}}}{\prod_{k=1}^{r} \delta_{G^{(k)}}^{(k)} \prod_{k=1}^{r} g_{k} !}
$$

Where $\phi$ and $\phi_{i}$ are defined by

$$
\phi=\frac{\prod_{j=1}^{n} \Gamma^{A_{j}}\left(1-a_{j}+\sum_{i=1}^{r} \alpha_{j}^{(i)} S_{k}\right)}{\prod_{j=n+1}^{p} \Gamma^{A_{j}}\left(a_{j}-\sum_{i=1}^{r} \alpha_{j}^{(i)} S_{k}\right) \prod_{j=1}^{q} \Gamma^{B_{j}}\left(1-b j+\sum_{i=1}^{r} \beta_{j}^{(i)} S_{k}\right)}
$$

and

$$
\phi_{i}=\frac{\prod_{j=1}^{n_{i}} \Gamma^{C_{j}^{(i)}}\left(1-c_{j}^{(i)}+\gamma_{j}^{(i)} S_{k}\right) \prod_{j=1}^{m_{i}} \Gamma\left(d_{j}^{(i)}-\delta_{j}^{(i)} S_{k}\right)}{\prod_{j=n_{i}+1}^{p_{i}} \Gamma^{C_{j}^{(i)}}\left(c_{j}^{(i)}-\gamma_{j}^{(i)} S_{k}\right) \prod_{j=m_{i}+1}^{q_{i}} \Gamma^{D_{j}^{(i)}}\left(1-d_{j}^{(i)}+\delta_{j}^{(i)} S_{k}\right)}, i=1, \cdots, r
$$

where

$$
S_{k}=\eta_{G_{k}, g_{k}}=\frac{d_{g_{k}}^{(k)}+G_{k}}{\delta_{g_{k}}^{(k)}} \text { for } k=1, \cdots, r
$$

which is valid under the following conditions: $\epsilon_{M_{k}}^{(k)}\left[p_{j}^{(k)}+p_{k}^{\prime}\right] \neq \epsilon_{j}^{(k)}\left[p_{M_{k}}+g_{k}\right]$

Consider the second multivariable I-function.

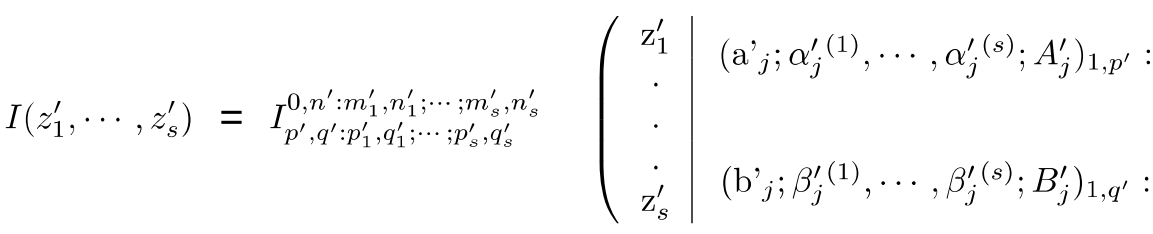

$$
\begin{aligned}
& \begin{array}{c}
\left(\mathrm{c}_{j}^{\prime(1)}, \gamma_{j}^{\prime(1)} ; C_{j}^{\prime(1)}\right)_{1, p_{1}^{\prime}} ; \cdots ;\left(c_{j}^{\prime(s)}, \gamma_{j}^{\prime(s)} ; C_{j}^{\prime(s)}\right)_{1, p_{s}^{\prime}} \\
\left(\mathrm{d}_{j}^{\prime(1)}, \delta_{j}^{\prime(1)} ; D_{j}^{\prime(1)}\right)_{1, q_{1}^{\prime}} ; \cdots ;\left(d_{j}^{\prime(s)}, \delta_{j}^{\prime(s)} ; D_{j}^{\prime(s)}\right)_{1, q_{s}^{\prime}}
\end{array}=\frac{1}{(2 \pi \omega)^{s}} \int_{L_{1}^{\prime}} \cdots \int_{L_{s}^{\prime}} \zeta\left(t_{1}, \cdots, t_{s}\right) \prod_{k=1}^{s} \phi_{k}\left(t_{k}\right) z_{k}^{\prime t_{k}} \mathrm{~d} t_{1} \cdots \mathrm{d} t_{s}
\end{aligned}
$$

where $\zeta\left(t_{1}, \cdots, t_{s}\right), \phi_{i}\left(s_{i}\right), i=1, \cdots, s$ are given by :

$$
\begin{aligned}
\zeta\left(t_{1}, \cdots, t_{s}\right) & =\frac{\prod_{j=1}^{n^{\prime}} \Gamma^{A_{j}^{\prime}}\left(1-a_{j}^{\prime}+\sum_{i=1}^{s} \alpha_{j}^{\prime(i)} t_{j}\right)}{\prod_{j=n^{\prime}+1}^{p^{\prime}} \Gamma^{A_{j}^{\prime}}\left(a_{j}^{\prime}-\sum_{i=1}^{s} \alpha_{j}^{\prime(i)} t_{j}\right) \prod_{j=1}^{q^{\prime}} \Gamma^{B_{j}^{\prime}}\left(1-b_{j}^{\prime}+\sum_{i=1}^{s} \beta_{j}^{\prime(i)} t_{j}\right)} \\
\phi_{i}\left(s_{i}\right) & =\frac{\prod_{j=1}^{n_{i}^{\prime}} \Gamma^{C_{j}^{\prime(i)}}\left(1-c_{j}^{\prime(i)}+\gamma_{j}^{\prime(i)} t_{i}\right) \prod_{j=1}^{m_{i}^{\prime}} \Gamma^{D_{j}^{\prime(i)}}\left(d_{j}^{\prime(i)}-\delta_{j}^{\prime(i)} t_{i}\right)}{\prod_{j=n_{i}^{\prime}+1}^{p_{i}^{\prime}} \Gamma^{C_{j}^{\prime(i)}}\left(c_{j}^{(i)}-\gamma_{j}^{\prime(i)} t_{i}\right) \prod_{j=m_{i}^{\prime}+1}^{q_{i}^{\prime}} \Gamma^{D_{j}^{\prime(i)}}\left(1-d_{j}^{\prime(i)}-\delta_{j}^{\prime(i)} t_{i}\right)}
\end{aligned}
$$

For more details, see Prathima et al. [6].

Following the result of Braaksma [3], the I-function of $r$ variables is analytic if: 


$$
U_{i}^{\prime}=\sum_{j=1}^{p^{\prime}} A_{j}^{\prime} \alpha_{j}^{\prime(i)}-\sum_{j=1}^{q^{\prime}} B_{j}^{\prime} \beta_{j}^{\prime(i)}+\sum_{j=1}^{p_{i}^{\prime}} C_{j}^{(i)} \gamma_{j}^{\prime(i)}-\sum_{j=1}^{q_{i}^{\prime}} D_{j}^{\prime(i)} \delta_{j}^{\prime(i)} \leqslant 0 ; i=1, \cdots, s
$$

The integral (1.13) converges absolutely if $\left|\arg \left(z_{k}^{\prime}\right)\right|<\frac{1}{2} \Delta_{k}^{\prime} \pi ; k=1, \cdots, s$. Where

$$
\Delta_{k}^{\prime}=-\sum_{j=n^{\prime}+1}^{p^{\prime}} A_{j}^{\prime} \alpha_{j}^{\prime(k)}-\sum_{j=1}^{q^{\prime}} B_{j}^{\prime} \beta_{j}^{\prime(k)}+\sum_{j=1}^{m_{k}^{\prime}} D_{j}^{\prime(k)} \delta_{j}^{\prime(k)}-\sum_{j=m_{k}^{\prime}+1}^{q_{k}^{\prime}} D_{j}^{\prime(k)} \delta_{j}^{\prime(k)}+\sum_{j=1}^{n_{k}^{\prime}} C_{j}^{\prime(k)} \gamma_{j}^{\prime(k)}-\sum_{j=n_{k}^{\prime}+1}^{p_{k}^{\prime}} C_{j}^{\prime(k)} \gamma_{j}^{\prime(k)}>0
$$

\section{Integral Representation of Generalized Hypergeometric Function}

The following generalized hypergeometric function regarding multiple integrals contour is also required [15, page 39 eq .30]

$$
\begin{aligned}
& \frac{\prod_{j=1}^{P} \Gamma\left(A_{j}\right)}{\prod_{j=1}^{Q} \Gamma\left(B_{j}\right)} P F_{Q}\left[\left(A_{P}\right) ;\left(B_{Q}\right) ;-\left(x_{1}+\cdots+x_{r}\right)\right] \\
& =\frac{1}{(2 \pi \omega)^{r}} \int_{L_{1}} \cdots \int_{L_{r}} \frac{\prod_{j=1}^{P} \Gamma\left(A_{j}+s_{1}+\cdots+s_{r}\right)}{\prod_{j=1}^{Q} \Gamma\left(B_{j}+s_{1}+\cdots+s_{r}\right)} \Gamma\left(-s_{1}\right) \cdots \Gamma\left(-s_{r}\right) x_{1}^{s_{1}} \cdots x_{r}^{s_{r}} \mathrm{~d} s_{1} \cdots \mathrm{d} s_{r}
\end{aligned}
$$

where the contours are of Barnes type with indentations, if necessary, to ensure that the poles of $\Gamma\left(A_{j}+s_{1}+\cdots+s_{r}\right)$ are separated from those of $\Gamma\left(-s_{j}\right), j=1, \cdots, r$. The above result (2.1) is easily established by an appeal to the calculus of residues by calculating the residues at the poles of $\Gamma\left(-s_{j}\right), j=1, \cdots, r$

The equivalent form of Eulerian beta integral is given by (1.1):

\section{MAIN INTEGRAL}

We shall note:

$X=m_{1}^{\prime}, n_{1}^{\prime} ; \cdots ; m_{s}^{\prime}, n_{s}^{\prime} ; 1,0 ; \cdots ; 1,0 ; 1,0 ; \cdots ; 1,0$

$Y=p_{1}^{\prime}, q_{1}^{\prime} ; \cdots ; p_{s}^{\prime}, q_{s}^{\prime} ; 0,1 ; \cdots ; 0,1 ; 0,1 ; \cdots ; 0,1$

$\mathbb{A}=\left[1+\sigma_{i}^{(1)}-\sum_{k^{\prime}=1}^{v} K_{k^{\prime}} \rho_{i}^{\prime \prime\left(1, k^{\prime}\right)}-\sum_{k=1}^{r} \eta_{G_{k}, g_{k}} \rho_{i}^{(1, k)}-\theta_{i}^{(1)}\left(2 R+k^{\prime}\right) ; \rho_{i}^{\prime(1,1)}, \cdots, \rho_{i}^{(1, s)}, \tau_{i}^{(1,1)}, \cdots, \tau_{i}^{(1, l)}, 1,0, \cdots, 0 ; 1\right]_{1, s}$

$, \cdots,\left[1+\sigma_{i}^{(T)}-\sum_{k^{\prime}=1}^{v} K_{k^{\prime}} \rho_{i}^{\prime \prime\left(T, k^{\prime}\right)}-\sum_{k=1}^{r} \eta_{G_{k}, g_{k}} \rho_{i}^{(T, k)}-\theta_{i}^{(T)}\left(2 R+k^{\prime}\right) ; \rho_{i}^{\prime(T, 1)}, \cdots, \rho_{i}^{\prime(T, s)}, \tau_{i}^{(T, 1)}, \cdots, \tau_{i}^{(T, l)}, 1,0, \cdots, 0 ; 1\right]_{1, s}$,

$\left[1-A_{j} ; 0, \cdots, 0,1, \cdots, 1,0, \cdots, 0 ; 1\right]_{1, P}$

$\left[1-\alpha_{i}-\sum_{k^{\prime}=1}^{v} K_{k^{\prime}} \delta_{i}^{\prime \prime\left(k^{\prime}\right)}-\sum_{k=1}^{r} \eta_{G_{k}, g_{k}} \delta_{i}^{(k)}-\left(2 R+k^{\prime}\right) \zeta_{i} ; \delta_{i}^{\prime(1)}, \cdots, \delta_{i}^{\prime(s)}, \mu_{i}^{(1)}, \cdots, \mu_{i}^{(l)}, 1, \cdots, 1,0, \cdots, 0 ; 1\right]_{1, s}$ 


$$
\begin{aligned}
& {\left[1-\beta_{i}-\sum_{k^{\prime}=1}^{v} K_{k^{\prime}} \eta_{i}^{\prime \prime\left(k^{\prime}\right)}-\sum_{k=1}^{r} \eta_{G_{k}, g_{k}} \eta_{i}^{(k)}-\left(2 R+k^{\prime}\right) \lambda_{i} ; \eta_{i}^{(1)}, \cdots, \eta_{i}^{\prime(s)}, \theta_{i}^{(1)}, \cdots, \theta_{i}^{(l)}, 1, \cdots, 1,0, \cdots, 0 ; 1\right]_{1, s}} \\
& \mathbf{A}=\left(a_{j}^{\prime} ; \alpha_{j}^{\prime(1)}, \cdots, \alpha_{j}^{\prime(s)}, 0, \cdots, 0,0, \cdots, 0 ; A_{j}^{\prime}\right)_{1, p^{\prime}}:\left(c_{j}^{\prime(1)}, \gamma_{j}^{\prime(1)} ; C_{j}^{\prime(1)}\right)_{1, p_{1}^{\prime}} ; \cdots ;\left(c_{j}^{\prime(r)}, \gamma_{j}^{(s)} ; C_{j}^{\prime(s)}\right)_{1, p_{s}^{\prime}} ;
\end{aligned}
$$$$
(1,0 ; 1) ; \cdots ;(1,0 ; 1) ;(1,0 ; 1) ; \cdots ;(1,0 ; 1)
$$$$
\mathbb{B}=\left[1+\sigma_{i}^{(1)}-\sum_{k^{\prime}=1}^{v} K_{k^{\prime}} \rho_{i}^{\prime \prime\left(1, k^{\prime}\right)}-\sum_{k=1}^{r} \eta_{G_{k}, g_{k}} \rho_{i}^{(1, k)}-\theta_{i}^{(1)}\left(2 R+k^{\prime}\right) ; \rho_{i}^{\prime(1,1)}, \cdots, \rho_{i}^{\prime(1, s)}, \tau_{i}^{(1,1)}, \cdots, \tau_{i}^{(1, l)}, 0, \cdots, 0 ; 1\right]_{1, s}
$$$$
, \cdots,\left[1+\sigma_{i}^{(T)}-\sum_{k^{\prime}=1}^{v} K_{k^{\prime}} \rho_{i}^{\prime \prime\left(T, k^{\prime}\right)}-\sum_{k=1}^{r} \eta_{G_{k}, g_{k}} \rho_{i}^{(T, k)}-\theta_{i}^{(T)}\left(2 R+k^{\prime}\right) ; \rho_{i}^{\prime(T, 1)}, \cdots, \rho_{i}^{\prime(T, s)}, \tau_{i}^{(T, 1)}, \cdots, \tau_{i}^{(T, l)}, 0, \cdots, 0 ; 1\right]_{1, s}
$$

$\left[1-B_{j} ; 0, \cdots, 0,1, \cdots, 1,0, \cdots, 0\right]_{1, Q}$

$\left[1-\alpha_{i}-\beta_{i}-\sum_{k^{\prime}=1}^{v}\left(\delta_{i}^{\prime \prime\left(k^{\prime}\right)}+\eta_{i}^{\prime \prime\left(k^{\prime}\right)}\right) K_{k^{\prime}}-\sum_{k=1}^{r}\left(\delta_{i}^{(k)}+\eta_{i}^{(k)}\right) \eta_{G_{k}, g_{k}}-\left(\zeta_{i}+\lambda_{i}\right)\left(2 R+k^{\prime}\right)\right.$

$$
\left.\left(\delta_{i}^{\prime(1)}+\eta_{i}^{\prime(1)}\right), \cdots,\left(\delta_{i}^{\prime(s)}+\eta_{i}^{\prime(s)}\right),\left(\mu_{i}^{(1)}+\theta_{i}^{(1)}\right), \cdots,\left(\mu_{i}^{(l)}+\theta_{i}^{(l)}\right), 1, \cdots, 1 ; 1\right]_{1, s}
$$

$\mathbf{B}=\left(b_{j}^{\prime} ; \beta_{j}^{\prime(1)}, \cdots, \beta_{j}^{\prime(s)}, 0, \cdots, 0,0, \cdots, 0 ; B_{j}^{\prime}\right)_{1, q^{\prime}}:\left(\mathrm{d}_{j}^{(1)}, \delta_{j}^{\prime(1)} ; D_{j}^{\prime(1)}\right)_{1, q_{1}^{\prime}} ; \cdots ;\left(d_{j}^{\prime(s)}, \delta_{j}^{\prime(s)} ; D_{j}^{\prime(s)}\right)_{1, q_{s}^{\prime}}$

$(0,1 ; 1) ; \cdots ;(0,1 ; 1) ;(0,1 ; 1) ; \cdots ;(0,1 ; 1)$

We have the following multiple Eulerian integrals, and we obtain the I-function of variables.

\section{Theorem}

$$
\begin{aligned}
& \int_{u_{1}}^{v_{1}} \cdots \int_{u_{t}}^{v_{t}} \prod_{i=1}^{t}\left[\left(x_{i}-u_{i}\right)^{\alpha_{i}-1}\left(v_{i}-x_{i}\right)^{\beta_{i}-1} \prod_{j=1}^{T}\left(U_{i}^{(j)} x_{i}+V_{i}^{(j)}\right)^{\sigma_{i}^{(j)}}\right] \\
& \left.\begin{array}{c}
\bar{I}\left(\begin{array}{c}
\mathrm{z}_{1} \prod_{i=1}^{t}\left[\frac{\left(x_{i}-u_{i}\right)^{\delta_{i}^{(1)}}\left(v_{i}-x_{i}\right)^{\eta_{i}^{(1)}}}{\prod_{j=1}^{T}\left(U_{i}^{(j)} x_{i}+V_{i}^{(j)}\right)^{\rho_{i}^{(j, 1)}}}\right] \\
\cdot \\
\cdot \\
\cdot \\
\mathrm{z}_{r} \prod_{i=1}^{t}\left[\begin{array}{c}
\left(x_{i}-u_{i}\right)_{i}^{\delta_{i}^{(r)}}\left(v_{i}-x_{i}\right)^{\eta_{i}^{(r)}} \\
\prod_{j=1}^{T}\left(U_{i}^{(j)} x_{i}+V_{i}^{(j)}\right)^{\rho_{i}^{(j, r)}}
\end{array}\right]
\end{array}\right)
\end{array}\right)\left(\begin{array}{c}
\mathrm{z}_{1}^{\prime} \prod_{i=1}^{t}\left[\frac{\left(x_{i}-u_{i}\right)^{\delta_{i}^{\prime(1)}}\left(v_{i}-x_{i}\right)^{\eta_{i}^{\prime(1)}}}{\prod_{j=1}^{T}\left(U_{i}^{(j)} x_{i}+V_{i}^{(j)}\right)^{\rho_{i}^{(j, 1)}}}\right] \\
\cdot \\
\cdot \\
\cdot \\
\mathrm{z}_{s}^{\prime} \prod_{i=1}^{t}\left[\frac{\left(x_{i}-u_{i}\right)^{\delta_{i}^{\prime(s)}}\left(v_{i}-x_{i}\right)^{\eta_{i}^{\prime(s)}}}{\prod_{j=1}^{T}\left(U_{i}^{(j)} x_{i}+V_{i}^{(j)}\right)^{\rho_{i}^{(j, s)}}}\right]
\end{array}\right)
\end{aligned}
$$




$$
S_{N_{1}, \cdots, N_{v}}^{\mathfrak{M}_{1}, \cdots, \mathfrak{M}_{\mathfrak{v}}}\left(\begin{array}{c}
\mathrm{z}_{1}^{\prime \prime} \prod_{i=1}^{t}\left[\frac{\left(x_{i}-u_{i}\right)^{\delta_{i}^{\prime \prime(1)}}\left(v_{i}-x_{i}\right)^{\eta_{i}^{\prime \prime(1)}}}{\prod_{j=1}^{T}\left(U_{i}^{(j)} x_{i}+V_{i}^{(j)}\right)^{\rho_{i}^{\prime \prime(j, 1)}}}\right] \\
\cdot \\
\cdot \\
\cdot \\
\mathrm{z}_{v}^{\prime \prime} \prod_{i=1}^{t}\left[\frac{\left(x_{i}-u_{i}\right)^{\delta_{i}^{\prime \prime(v)}}\left(v_{i}-x_{i}\right)^{\eta_{i}^{\prime \prime(v)}}}{\prod_{j=1}^{T}\left(U_{i}^{(j)} x_{i}+V_{i}^{(j)}\right)^{\rho_{i}^{\prime \prime(j, v)}}}\right]
\end{array}\right] \psi_{\alpha n^{\prime \prime}}\left[c^{\sigma}, \prod_{j=1}^{t}\left[\frac{\left(x_{i}-u_{i}\right)^{\zeta_{i}}\left(v_{i}-x_{i}\right)^{\lambda_{i}}}{\prod_{j=1}^{T}\left(U_{i}^{(j)} x_{i}+V_{i}^{(j)}\right)^{\theta_{i}^{(j)}}}\right]\right]
$$

$\underset{\text { ते }}{\stackrel{\infty}{\overrightarrow{5}}} \quad{ }_{P} F_{Q}\left[\left(A_{P}\right) ;\left(B_{Q}\right) ;-\sum_{k=1}^{l} g_{k} \prod_{i=1}^{t}\left[\frac{\left(x_{i}-u_{i}\right)^{u_{i}^{(k)}}\left(v_{i}-x_{i}\right)^{\theta_{i}^{(r)}}}{\prod_{j=1}^{T}\left(U_{i}^{(j)} x_{i}+V_{i}^{(j)}\right)^{\tau_{i}^{(j, k)}}}\right]\right] \mathrm{d} x_{1} \cdots \mathrm{d} x_{s}$

6

$$
=\frac{\prod_{j=1}^{Q} \Gamma\left(B_{j}\right)}{\prod_{j=1}^{P} \Gamma\left(A_{j}\right)} \prod_{j=1}^{t}\left[\left(v_{i}-u_{i}\right)^{\alpha_{i}+\beta_{i}-1} \prod_{j=1}^{W}\left(u_{i} U_{i}^{(j)}+V_{i}^{(j)}\right)^{\sigma_{i}^{(j)}} \prod_{j=W+1}^{T}\left(u_{i} U_{i}^{(j)}+V_{i}^{(j)}\right)^{\sigma_{i}^{(j)}}\right]
$$

$\sum_{k^{\prime \prime}=0, \text { or } 1}^{\infty_{*}} \sum_{R=0}^{\infty} \sum_{K_{1}=0}^{\left[N_{1} / \mathfrak{M}_{\mathbf{1}}\right]} \cdots \sum_{K_{v}=0}^{\left[N_{v} / \mathfrak{M}_{\mathfrak{v}}\right]} \sum_{G_{k}=1}^{m_{k}} \sum_{g_{k}=0}^{\infty} \phi \frac{\prod_{k=1}^{r} \phi_{k} z_{k}^{\eta_{G_{k}, g_{k}}}(-)^{\sum_{k=1}^{r} g_{k}}}{\prod_{k=1}^{r} \delta_{G^{(k)}}^{(k)} \prod_{k=1}^{r} g_{k} !} a_{v}^{\prime} z_{1}^{\prime \prime K_{1}} \cdots z_{v}^{\prime \prime K_{v}} \frac{(-)^{R} a_{k^{\prime \prime}}\left(c^{\sigma} \mid \alpha n^{\prime \prime}\right)}{R ! \Gamma\left(R+k^{\prime \prime}+\alpha+\frac{3}{2}\right)} E_{i j}$

$$
I_{s T+P+2 s+p^{\prime}, s T+Q+s+q^{\prime}: Y}^{0, s T+P+2 s+n^{\prime}: X}\left(\begin{array}{c|c}
\mathrm{z}_{1}^{\prime} w_{1} & \mathbb{A}, \mathbf{A} \\
\cdot & \cdot \\
\cdot & \cdot \\
\mathrm{z}_{1}^{\prime} w_{s} & \cdot \\
\mathrm{g}_{1} W_{1} & \cdot \\
\cdot & \cdot \\
\mathrm{g}_{l} W_{l} & \cdot \\
\mathrm{G}_{1} & \cdot \\
\cdot & \cdot \\
\cdot & \mathbb{B}, \mathbf{B}
\end{array}\right)
$$

\section{Where}

$$
\begin{gathered}
E_{i j}=\frac{\frac{i^{n^{\prime \prime}} \sqrt{2 \pi}}{V_{a n^{\prime \prime}(c)}}}{\prod_{i=1}^{t} \prod_{j=1}^{W}\left(u_{i} U_{i}^{(j)}+V_{i}^{(j)}\right)^{\sum_{k^{\prime}=1}^{v} \rho_{i}^{\prime \prime\left(j, k^{\prime}\right)} K_{k^{\prime}}+\sum_{k=1}^{r} \rho_{i}^{(j, k)} \eta_{G_{k}, g_{k}}+\theta_{i}^{(j)}\left(2 R+k^{\prime \prime}\right)}} \\
\times \frac{\left.\prod_{i=1}^{t}\left(v_{i}-u_{i}\right)^{\sum_{k^{\prime}=1}^{v}\left(\delta_{i}^{\prime \prime\left(k^{\prime}\right)}+\eta_{i}^{\prime \prime}\left(k^{\prime}\right)\right.}\right) K_{k^{\prime}}+\sum_{k=1}^{r}\left(\delta_{i}^{(k)}+\eta_{i}^{(k)}\right) \eta_{G_{k}, g_{k}}+\left(\zeta_{i}+\lambda_{i}\right)\left(2 R+k^{\prime \prime}\right)}{\prod_{i=1}^{t} \prod_{j=W+1}^{T}\left(u_{i} U_{i}^{(j)}+V_{i}^{(j)}\right)^{\sum_{k^{\prime}=1}^{v} \rho_{i}^{\prime \prime\left(j, k^{\prime}\right)} K_{k^{\prime}}+\sum_{k=1}^{r} \rho_{i}^{(j, k)} \eta_{G_{k}, g_{k}}+\theta_{i}^{(j)}\left(2 R+k^{\prime \prime}\right)}} \\
w_{m}=\prod_{i=1}^{t}\left[\left(v_{i}-u_{i}\right)^{\delta_{i}^{\prime(m)}+\eta_{i}^{\prime(m)}} \prod_{j=1}^{W}\left(u_{i} U_{i}^{(j)}+V_{i}^{(j)}\right)^{-\rho_{i}^{\prime(j, m)}} \prod_{j=W+1}^{T}\left(u_{i} U_{i}^{(j)}+V_{i}^{(j)}\right)^{\rho_{i}^{(j, m)}}\right], m=1, \cdots, s
\end{gathered}
$$




$$
\begin{aligned}
& W_{k}=\prod_{i=1}^{t}\left[\left(v_{i}-u_{i}\right)^{\mu_{i}^{(k)}+\theta_{i}^{(k)}} \prod_{j=1}^{W}\left(u_{i} U_{i}^{(j)}+V_{i}^{(j)}\right)^{-\tau_{i}^{(j, k)}} \prod_{j=W+1}^{T}\left(u_{i} U_{i}^{(j)}+V_{i}^{(j)}\right)^{-\tau_{i}^{(j, k)}}\right], k=1, \cdots, l \\
& G_{j}=\prod_{i=1}^{t}\left[\frac{\left(v_{i}-u_{i}\right) U_{i}^{(j)}}{u_{i} U_{i}^{(j)}+V_{i}^{(j)}}\right], j=1, \cdots, W
\end{aligned}
$$$$
G_{j}=-\prod_{i=1}^{t}\left[\frac{\left(v_{i}-u_{i}\right) U_{i}^{(j)}}{u_{i} U_{i}^{(j)}+V_{i}^{(j)}}\right], j=W+1, \cdots, T
$$$$
\sum_{G_{k}=1}^{m_{k}} \sum_{g_{k}=0}^{\infty}=\sum_{G_{1}, \cdots, G_{r}=1}^{m_{1}, \cdots, m_{r}} \sum_{g_{1}, \cdots, g_{r}=0}^{\infty}
$$

\section{Provided that:}

(A) $W \in[0, T] ; u_{i}, v_{i} \in \mathbb{R} ; i=1, \cdots, t$

(B) $\min \left\{\delta_{i}^{(g)}, \eta_{i}^{(g)}, \delta_{i}^{\prime(h)}, \eta_{i}^{\prime(h)}, \delta_{i}^{\prime \prime(k)}, \eta_{i}^{\prime \prime(k)}, \zeta_{i}, \eta_{i}\right\} \geqslant 0 ; g=1, \cdots, r ; i=1, \cdots, t ; h=1, \cdots, s ; k=1, \cdots, v$

$\min \left\{\rho_{i}^{(j, g)}, \rho_{i}^{\prime(j, h)}, \rho_{i}^{\prime \prime\left(j, k^{\prime}\right)}, \theta_{i}^{(j)}, \tau_{i}^{(j, k)}\right\} \geqslant 0 ; j=1, \cdots, T ; i=1, \cdots, t ; g=1, \cdots, r ; h=1, \cdots, s ; k^{\prime}=1, \cdots, v, k=1, \cdots, l$

(C) $\sigma_{i}^{(j)} \in \mathbb{R}, U_{i}^{(j)}, V_{i}^{(j)} \in \mathbb{C}, z_{i^{\prime}}, z_{j^{\prime}}, z_{k^{\prime}}^{\prime \prime}, g_{k}, G_{j} \in \mathbb{C} ; i=1, \cdots, t ; j=1, \cdots, T ; i^{\prime}=1, \cdots, r$;

$j^{\prime}=1, \cdots, s ; k^{\prime}=1, \cdots, v ; k=1, \cdots, l$

(D) $\max \left[\left|\frac{\left(v_{i}-u_{i}\right) U_{i}^{(j)}}{u_{i} U_{i}^{(j)}+V_{i}^{(j)}}\right|\right]<1, i=1, \cdots, s ; j=1, \cdots, W$ and

$\max \left[\left|\frac{\left(v_{i}-u_{i}\right) U_{i}^{(j)}}{u_{i} U_{i}^{(j)}+V_{i}^{(j)}}\right|\right]<1, i=1, \cdots, s ; j=W+1, \cdots, T$

(E) $\left|\arg \left(z_{i} \prod_{j=1}^{T}\left(U_{i}^{(j)} x_{i}+V_{i}^{(j)}\right)^{\rho_{i}^{(j, k)}}\right)\right|<\frac{1}{2} \Delta_{k} \pi, k=1, \cdots, r$, where

$\Delta_{k}=-\sum_{j=n+1}^{p} A_{j} \alpha_{j}^{(k)}-\sum_{j=1}^{q} B_{j} \beta_{j}^{(k)}+\sum_{j=1}^{m_{k}} \delta_{j}^{(k)}-\sum_{j=m_{k}+1}^{q_{k}} D_{j}^{(k)} \delta_{j}^{(k)}+\sum_{j=1}^{n_{k}} C_{j}^{(k)} \gamma_{j}^{(k)}-\sum_{j=n_{k}+1}^{p_{k}} C_{j}^{(k)} \gamma_{j}^{(k)}$

$-\delta_{i}^{(k)}-\eta_{i}^{(k)}-\sum_{j=1}^{T} \rho_{i}^{(j, k)}>0$

$\left|\arg \left(z_{i}^{\prime} \prod_{j=1}^{T}\left(U_{i}^{(j)} x_{i}+V_{i}^{(j)}\right)^{\rho_{i}^{\prime(j, k)}}\right)\right| \frac{1}{2} \Delta_{k}^{\prime} \pi, k=1, \cdots, s$, where

$\Delta_{k}^{\prime}=-\sum_{j=n^{\prime}+1}^{p^{\prime}} A_{j}^{\prime} \alpha_{j}^{\prime(k)}-\sum_{j=1}^{q^{\prime}} B_{j}^{\prime} \beta_{j}^{\prime(k)}+\sum_{j=1}^{m_{k}^{\prime}} D_{j}^{\prime(k)} \delta_{j}^{\prime(k)}-\sum_{j=m_{k}^{\prime}+1}^{q_{k}^{\prime}} D_{j}^{\prime(k)} \delta_{j}^{\prime(k)}+\sum_{j=1}^{n_{k}^{\prime}} C_{j}^{\prime(k)} \gamma_{j}^{\prime(k)}-\sum_{j=n_{k}^{\prime}+1}^{p_{k}^{\prime}} C_{j}^{\prime(k)} \gamma_{j}^{\prime(k)}$

$-\delta_{i}^{\prime(k)}-\eta_{i}^{\prime(k)}-\sum_{j=1}^{T} \rho_{i}^{\prime(j, k)}>0$ 
(F) $\operatorname{Re}\left(\alpha_{i}+\zeta_{i}\left(2 R+k^{\prime \prime}\right)+\sum_{j=1}^{r} \delta_{i}^{(j)} \eta_{G_{j}, g_{j}}\right)+\sum_{k=1}^{s} \delta_{i}^{\prime(k)} \min _{1 \leqslant j \leqslant m_{k}^{\prime}} R e\left(\frac{d_{j}^{\prime(k)}}{\delta_{j}^{\prime(k)}}\right)>0$ and

$$
\operatorname{Re}\left(\beta_{i}+\lambda_{i}\left(2 R+k^{\prime \prime}\right)+\sum_{j=1}^{r} \eta_{i}^{(j)} \eta_{G_{j}, g_{j}}\right)+\sum_{k=1}^{s} \eta_{i}^{\prime(k)} \min _{1 \leqslant j \leqslant m_{k}^{\prime}} \operatorname{Re}\left(\frac{d_{j}^{(k)}}{\delta_{j}^{\prime(k)}}\right)>0 \text { for } i=1, \cdots, t
$$

(G) $P \leqslant Q+1$. The equality holds, when, in addition,

$\underset{\stackrel{\infty}{*}}{\stackrel{\infty}{*}}$ either $P>Q$ and $\sum_{k=1}^{l}\left|g_{k}\left(\prod_{j=1}^{T}\left(U_{i}^{(j)} x_{i}+V_{i}^{(j)}\right)^{\tau_{i}^{(j, k)}}\right)\right|^{\frac{1}{Q-P}}<1 \quad\left(u_{i} \leqslant x_{i} \leqslant v_{i} ; i=1, \cdots, t\right)$

or $P \leqslant Q$ and $\max _{1 \leqslant k \leqslant l}\left[\left|\left(g_{k} \prod_{j=1}^{T}\left(U_{i}^{(j)} x_{i}+V_{i}^{(j)}\right)^{-\tau_{i}^{(j, k)}}\right)\right|\right]<1 \quad\left(u_{i} \leqslant x_{i} \leqslant v_{i} ; i=1, \cdots, t\right)$

\section{Proof}

To establish the formula (4.7), we first express the spheroidal function, the class of multivariable polynomials $S_{N_{1}, \cdots, N_{v}}^{\mathfrak{M}_{1}, \cdots, \mathfrak{M}_{\mathfrak{v}}}[$.$] and the multivariable I-function \bar{I}\left(z_{1}, \cdots, z_{r}\right)$ in series with the help of $(1,2),(1,3)$ and (1.7) respectively, use integral contour representation with the help of (1.8) for the multivariable I-function $I\left(z_{1}^{\prime}, \cdots, z_{s}^{\prime}\right)$ occurring in its left-hand side and use the integral contour representation with the help of (2.1) for the Generalized hypergeometric function ${ }_{P} F_{Q}($.$) . Changing the order of$ integration and summation (which is easily seen to be justified due to the absolute convergence of the integral and the summations involved in the process). Now we write:

$$
\prod_{j=1}^{T}\left(U_{i}^{(j)} x_{i}+V_{i}^{(j)}\right)^{K_{i}^{(j)}}=\prod_{j=1}^{W}\left(U_{i}^{(j)} x_{i}+V_{i}^{(j)}\right)^{K_{i}^{(j)}} \prod_{j=W+1}^{T}\left(U_{i}^{(j)} x_{i}+V_{i}^{(j)}\right)^{K_{i}^{(j)}}
$$

where $K_{i}^{(j)}=v_{i}^{(j)}-\theta_{i}^{(j)}\left(2 R+k^{\prime \prime}\right)-\sum_{l=1}^{r} \rho_{i}^{(j, l)} \eta_{G_{l}, g_{l}}-\sum_{l=1}^{s} \rho_{i}^{\prime(j, l)} \psi_{l}-\sum_{l=1}^{v} \rho_{i}^{\prime \prime(j, v)} K_{l}$ where $i=1, \cdots, t ; j=1, \cdots, T$

and express the factors occurring in R.H.S. Of (3.1) in terms of following Mellin-Barnes integrals contour, we obtain:

$$
\begin{aligned}
& \prod_{j=1}^{W}\left(U_{i}^{(j)} x_{i}+V_{i}^{(j)}\right)^{K_{i}^{(j)}}=\prod_{j=1}^{W}\left[\frac{\left(U_{i}^{(j)} u_{i}+V_{i}^{(j)}\right)^{K_{i}^{(j)}}}{\Gamma\left(-K_{i}^{(j)}\right)}\right] \frac{1}{(2 \pi \omega)^{W}} \int_{L_{1}^{\prime}} \cdots \int_{L_{W}^{\prime}} \prod_{j=1}^{W}\left[\Gamma\left(-\zeta_{j}^{\prime}\right) \Gamma\left(-K_{i}^{(j)}+\zeta_{j}^{\prime}\right)\right. \\
& \left.\prod_{j=1}^{W}\left[\frac{\left(U_{i}^{(j)}\left(x_{i}-u_{i}\right)\right.}{\left(u_{i} U_{i}^{(j)}+V_{i}^{(j)}\right)}\right]^{\zeta_{j}^{\prime}}\right] \mathrm{d} \zeta_{1}^{\prime} \cdots \mathrm{d} \zeta_{W}^{\prime}
\end{aligned}
$$

$\prod_{j=W+1}^{T}\left(U_{i}^{(j)} x_{i}+V_{i}^{(j)}\right)^{K_{i}^{(j)}}=\prod_{j=W+1}^{T}\left[\frac{\left(U_{i}^{(j)} v_{i}+V_{i}^{(j)}\right)^{K_{i}^{(j)}}}{\Gamma\left(-K_{i}^{(j)}\right)}\right] \frac{1}{(2 \pi \omega)^{T-W}} \int_{L_{W+1}^{\prime}} \ldots \int_{L_{T}^{\prime} j} \prod_{W+1}^{T}\left[\Gamma\left(-\zeta_{j}^{\prime}\right) \Gamma\left(-K_{i}^{(j)}+\zeta_{j}^{\prime}\right)\right.$

$\left.\prod_{j=W+1}^{T}\left[-\frac{\left(U_{i}^{(j)}\left(v_{i}-x_{i}\right)\right.}{\left(v_{i} U_{i}^{(j)}+V_{i}^{(j)}\right)}\right]^{\zeta_{j}^{\prime}}\right] \mathrm{d} \zeta_{W+1}^{\prime} \cdots \mathrm{d} \zeta_{T}^{\prime}$ 
We apply the Fubini's theorem for multiple integrals. Finally evaluating the innermost x-integral with the help of (1.1) and reinterpreting the multiple Mellin-Barnes integrals contour in terms of multivariable I-function of $(r+l+T)$ variables, we obtain the formula (3.1).

\section{Particular Cases}

\section{a) I-functions of two variables}

\section{Corollary 1}

If $r=s=2$, then the multivariable I-functions reduce to I-functions of two variables defined by Rathie et al. [9]. We have.

$$
\int_{u_{1}}^{v_{1}} \cdots \int_{u_{t}}^{v_{t}} \prod_{i=1}^{t}\left[\left(x_{i}-u_{i}\right)^{\alpha_{i}-1}\left(v_{i}-x_{i}\right)^{\beta_{i}-1} \prod_{j=1}^{T}\left(U_{i}^{(j)} x_{i}+V_{i}^{(j)}\right)^{\sigma_{i}^{(j)}}\right]
$$

$\left.\begin{array}{c}\mathrm{z}_{1} \prod_{i=1}^{t}\left[\frac{\left(x_{i}-u_{i}\right)_{i}^{\delta^{(1)}}\left(v_{i}-x_{i}\right)_{i}^{\eta_{i}^{(1)}}}{\prod_{j=1}^{T}\left(U_{i}^{(j)} x_{i}+V_{i}^{(j)}\right)^{\rho_{i}^{(j, 1)}}}\right] \\ \cdot \\ \cdot \\ \mathrm{z}_{2} \prod_{i=1}^{t}\left[\begin{array}{c}\left(x_{i}-u_{i}\right)_{i}^{\delta^{(2)}}\left(v_{i}-x_{i}\right)^{\eta_{i}^{(2)}} \\ \prod_{j=1}^{T}\left(U_{i}^{(j)} x_{i}+V_{i}^{(j)}\right)^{\rho_{i}^{(j, 2)}}\end{array}\right]\end{array}\right)\left(\begin{array}{c}\mathrm{z}_{1}^{\prime} \prod_{i=1}^{t}\left[\frac{\left(x_{i}-u_{i}\right)^{\delta_{i}^{\prime(1)}}\left(v_{i}-x_{i}\right)^{\eta_{i}^{\prime(1)}}}{\prod_{j=1}^{T}\left(U_{i}^{(j)} x_{i}+V_{i}^{(j)}\right)^{\rho_{i}^{\prime}(j, 1)}}\right] \\ \cdot \\ \cdot \\ \cdot \\ \mathrm{z}_{2}^{\prime} \prod_{i=1}^{t}\left[\frac{\left(x_{i}-u_{i}\right)^{\delta_{i}^{\prime(2)}}\left(v_{i}-x_{i}\right)^{\eta_{i}^{\prime(2)}}}{\prod_{j=1}^{T}\left(U_{i}^{(j)} x_{i}+V_{i}^{(j)}\right)^{\rho_{i}^{\prime}(j, 2)}}\right]\end{array}\right)$

$$
S_{N_{1}, \cdots, N_{v}}^{\mathfrak{M}_{1}, \cdots, \mathfrak{M}_{\mathfrak{v}}}\left(\begin{array}{c}
\mathrm{z}_{1}^{\prime \prime} \prod_{i=1}^{t}\left[\frac{\left(x_{i}-u_{i}\right)^{\delta_{i}^{\prime \prime(1)}}\left(v_{i}-x_{i}\right)^{\eta_{i}^{\prime \prime(1)}}}{\prod_{j=1}^{T}\left(U_{i}^{(j)} x_{i}+V_{i}^{(j)}\right)^{\rho_{i}^{\prime \prime(j, 1)}}}\right] \\
\cdot \\
\cdot \\
\cdot \\
\mathrm{z}_{v}^{\prime \prime} \prod_{i=1}^{t}\left[\frac{\left(x_{i}-u_{i}\right)^{\delta_{i}^{\prime \prime(v)}}\left(v_{i}-x_{i}\right)^{\eta_{i}^{\prime \prime(v)}}}{\prod_{j=1}^{T}\left(U_{i}^{(j)} x_{i}+V_{i}^{(j)}\right)^{\rho_{i}^{\prime \prime(j, v)}}}\right]
\end{array}\right] \psi_{\alpha n^{\prime \prime}}\left[c^{\sigma}, \prod_{j=1}^{t}\left[\frac{\left(x_{i}-u_{i}\right)^{\zeta_{i}}\left(v_{i}-x_{i}\right)^{\lambda_{i}}}{\prod_{j=1}^{T}\left(U_{i}^{(j)} x_{i}+V_{i}^{(j)}\right)^{\theta_{i}^{(j)}}}\right]\right]
$$

${ }_{P} F_{Q}\left[\left(A_{P}\right) ;\left(B_{Q}\right) ;-\sum_{k=1}^{l} g_{k} \prod_{i=1}^{t}\left[\frac{\left(x_{i}-u_{i}\right)^{u_{i}^{(k)}}\left(v_{i}-x_{i}\right)^{\theta_{i}^{(r)}}}{\prod_{j=1}^{T}\left(U_{i}^{(j)} x_{i}+V_{i}^{(j)}\right)^{\tau_{i}^{(j, k)}}}\right]\right] \mathrm{d} x_{1} \cdots \mathrm{d} x_{s}$

$$
=\frac{\prod_{j=1}^{Q} \Gamma\left(B_{j}\right)}{\prod_{j=1}^{P} \Gamma\left(A_{j}\right)} \prod_{j=1}^{t}\left[\left(v_{i}-u_{i}\right)^{\alpha_{i}+\beta_{i}-1} \prod_{j=1}^{W}\left(u_{i} U_{i}^{(j)}+V_{i}^{(j)}\right)^{\sigma_{i}^{(j)}} \prod_{j=W+1}^{T}\left(u_{i} U_{i}^{(j)}+V_{i}^{(j)}\right)^{\sigma_{i}^{(j)}}\right]
$$

$$
\sum_{k^{\prime \prime}=0, \text { or } 1}^{\infty} \sum_{R=0}^{\infty} \sum_{K_{1}=0}^{\left[N_{1} / \mathfrak{M}_{1}\right]} \cdots \sum_{K_{v}=0}^{\left[N_{v} / \mathfrak{M}_{\mathfrak{v}}\right]} \sum_{G_{1}=1}^{m_{1}} \sum_{G_{2}=1}^{m_{2}} \sum_{g_{1}, g_{2}=0}^{\infty} \phi_{2} \frac{\prod_{k=1}^{2} \phi_{2 k} z_{k}^{\eta_{G_{k}, g_{k}}}(-)^{\sum_{k=1}^{2} g_{k}}}{\prod_{k=1}^{2} \delta_{G^{(k)}}^{(k)} \prod_{k=1}^{2} g_{k} !} a_{v}^{\prime \prime} z_{1}^{\prime \prime K_{1}} \cdots z_{v}^{\prime \prime K_{v}} \frac{(-)^{R} a_{k^{\prime \prime}}\left(c^{\sigma} \mid \alpha n^{\prime \prime}\right)}{R ! \Gamma\left(R+k^{\prime \prime}+\alpha+\frac{3}{2}\right)}
$$




$$
E_{i j} I_{s T+P+2 s+p^{\prime}, s T+Q+X_{2}, s T+P+q^{\prime}: Y_{2}}\left(\begin{array}{c|c}
\mathrm{z}_{1}^{\prime} w_{1} & \mathbb{A}_{2}, \mathbf{A}_{2} \\
\cdot & \cdot \\
\cdot & \cdot \\
\mathrm{z}_{2}^{\prime} w_{2} & \cdot \\
\mathrm{g}_{1} W_{1} & ; \\
\cdot & \cdot \\
\cdot & \cdot \\
\mathrm{g}_{l} W_{l} & \cdot \\
\mathrm{G}_{1} & \cdot \\
\cdot & \cdot \\
\cdot & \mathbb{B}_{2}, \mathbf{B}_{2} \\
\mathrm{G}_{T} &
\end{array}\right)
$$

10 The validity conditions are the same that (3.1) with $r=s=2$. The quantities $\phi_{2}, \phi_{2 k}, V_{2}, W_{2}, \mathbb{A}_{2}, \mathbb{B}_{2}, \mathbf{A}_{2}, \mathbf{B}_{2}$ are equal to $\phi, \phi_{k}, V, W, \mathbb{A}, \mathbb{B}, \mathbf{A}, \mathbf{B}$ respectively for $r=s=2$.

\section{b) I-function of one variable}

\section{Corollary 2}

If $r=s=1$, the multivariable I-functions reduce to I-functions of one variable defined by Rathie [8]. We have

$$
\begin{aligned}
& \int_{u_{1}}^{v_{1}} \cdots \int_{u_{t}}^{v_{t}} \prod_{i=1}^{t}\left[\left(x_{i}-u_{i}\right)^{\alpha_{i}-1}\left(v_{i}-x_{i}\right)^{\beta_{i}-1} \prod_{j=1}^{T}\left(U_{i}^{(j)} x_{i}+V_{i}^{(j)}\right)^{\sigma_{i}^{(j)}}\right] \\
& I\left(\mathrm{z} \prod_{i=1}^{t}\left[\frac{\left(x_{i}-u_{i}\right)_{i}^{\delta_{i}^{(1)}}\left(v_{i}-x_{i}\right)^{\eta_{i}^{(1)}}}{\prod_{j=1}^{T}\left(U_{i}^{(j)} x_{i}+V_{i}^{(j)}\right)^{\rho_{i}^{(j, 1)}}}\right]\right) I\left(\mathrm{z}^{\prime} \prod_{i=1}^{t}\left[\frac{\left(x_{i}-u_{i}\right)^{\delta_{i}^{\prime(1)}}\left(v_{i}-x_{i}\right)^{\eta_{i}^{\prime(1)}}}{\prod_{j=1}^{T}\left(U_{i}^{(j)} x_{i}+V_{i}^{(j)}\right)^{\rho_{i}^{(j, 1)}}}\right]\right)
\end{aligned}
$$

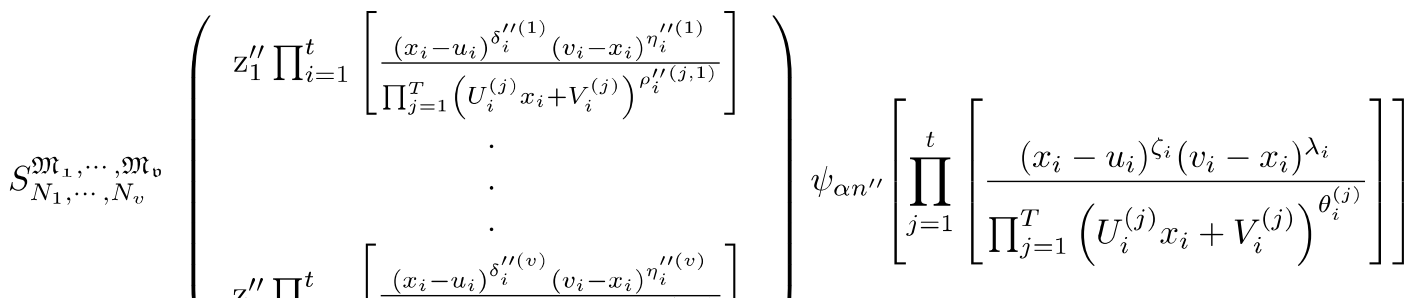

$$
\begin{aligned}
& \mathrm{z}_{v}^{\prime \prime} \prod_{i=1}^{t}\left[\frac{\left(x_{i}-u_{i}\right)^{\delta_{i}^{\prime \prime(v)}}\left(v_{i}-x_{i}\right)^{\eta_{i}^{\prime \prime(v)}}}{\prod_{j=1}^{T}\left(U_{i}^{(j)} x_{i}+V_{i}^{(j)}\right)^{\rho_{i}^{\prime(j, v)}}}\right]
\end{aligned}
$$$$
{ }_{P} F_{Q}\left[\left(A_{P}\right) ;\left(B_{Q}\right) ;-\sum_{k=1}^{l} g_{k} \prod_{i=1}^{t}\left[\frac{\left(x_{i}-u_{i}\right)^{u_{i}^{(k)}}\left(v_{i}-x_{i}\right)^{\theta_{i}^{(r)}}}{\prod_{j=1}^{T}\left(U_{i}^{(j)} x_{i}+V_{i}^{(j)}\right)^{\tau_{i}^{(j, k)}}}\right]\right] \mathrm{d} x_{1} \cdots \mathrm{d} x_{s}
$$$$
=\frac{\prod_{j=1}^{Q} \Gamma\left(B_{j}\right)}{\prod_{j=1}^{P} \Gamma\left(A_{j}\right)} \prod_{j=1}^{t}\left[\left(v_{i}-u_{i}\right)^{\alpha_{i}+\beta_{i}-1} \prod_{j=1}^{W}\left(u_{i} U_{i}^{(j)}+V_{i}^{(j)}\right)^{\sigma_{i}^{(j)}} \prod_{j=W+1}^{T}\left(u_{i} U_{i}^{(j)}+V_{i}^{(j)}\right)^{\sigma_{i}^{(j)}}\right]
$$

$$
\sum_{k^{\prime \prime}=0, \text { or } 1}^{\infty_{*}} \sum_{R=0}^{\infty} \sum_{K_{1}=0}^{\left[N_{1} / \mathfrak{M}_{1}\right]} \cdots \sum_{K_{v}=0}^{\left[N_{v} / \mathfrak{M}_{\mathfrak{v}}\right]} \sum_{G_{1}=1}^{m_{1}} \sum_{g_{1}=0}^{\infty} \phi_{1} \frac{z_{k}^{\eta_{G_{1}, g_{1}}}(-)^{g_{1}}}{\delta_{G^{(1)}}^{(1)} g_{1} !} a_{v}^{\prime} z_{1}^{\prime \prime K_{1}} \cdots z_{v}^{\prime \prime K_{v}} \frac{(-)^{R} a_{k^{\prime \prime}}\left(c^{\sigma} \mid \alpha n^{\prime \prime}\right)}{R ! \Gamma\left(R+k^{\prime \prime}+\alpha+\frac{3}{2}\right)} E_{i j}
$$




$$
I_{s T+P+2 s+p^{\prime}, s T+Q+X_{1}}^{0, s T+P+2 s+n^{\prime}: X_{1}: Y_{1}}\left(\begin{array}{c|c}
\mathrm{z}^{\prime} \mathrm{w}_{1} & \mathbb{A}_{1}, \mathbf{A}_{1} \\
\mathrm{~g}_{1} W_{1} & \cdot \\
\cdot & \cdot \\
\cdot & \cdot \\
\mathrm{g}_{l} W_{l} & \cdot \\
\mathrm{G}_{1} & \cdot \\
\cdot & \cdot \\
\cdot & \mathbb{B}_{1}, \mathbf{B}_{1}
\end{array}\right)
$$

The validity conditions are the same that (3.1) with $r=s=1$. The quantities $\phi_{1}, V_{1}, W_{1}, \mathbb{A}_{1}, \mathbb{B}_{1}, \mathbf{A}_{1}, \mathbf{B}_{1}$ are equal to $\phi_{k}, V, W, \mathbb{A}, \mathbb{B}, \mathbf{A}, \mathbf{B}$ respectively for $r=s=1$.

Remark: By the similar procedure, the results of this document can be extended to the product of any finite number of multivariable I-functions and class of multivariable polynomials defined by Srivastava [12].

\section{Conclusion}

Our main integral formula is unified in nature and possesses manifold generality. It acts a fundamental expression and using various particular cases of the multivariable I-function, the class of multivariable polynomials and a general spheroidal functions, one can obtain a large number of other integrals involving simpler special functions and polynomials of one and several variables.

\section{References Références Referencias}

1. F. Ayant, On general multiple Eulerian integrals involving the multivariable Ifunction, a general class of polynomials and the extension of Zeta-function, Int Jr. of Mathematical Sciences \& Applications, 6(2), (2016), 1051-1069.

2. A. Bhargava, A. Srivastava and O. Mukherjee, On a General Class of Multiple Eulerian Integrals. International Journal of Latest Technology in Engineering, Management \& Applied Science (IJLTEMAS), 3(8) (2014), 57-64.

3. B. L. J. Braaksma, "Asymptotic expansions and analytic continuations for a class of Barnes integrals," Composition Mathematical, vol. 15, pp. 239-341, 1964.

4. S.P. Goyal and T. Mathur, On general multiple Eulerian integrals and fractional integration, Vijnana Parishad Anusandhan 46(3) (2003), 231-246.

5. I.S. Gradsteyn and I.M. Ryxhik, Table of integrals, series and products: Academic press, New York 1980.

6. J. Prathima, V. Nambisan and S.K.Kurumujji, A Study of I-function of Several Complex Variables, International Journal of Engineering Mathematics Vol(2014), 2014, 1-12.

7. R. K. Raina, R. K. and H. M. Srivastava, Evaluation of certain class of Eulerian integrals. J. phys. A: Math.Gen. 26(1993), 691-696.

8. A.K. Rathie, A new generalization of generalized hypergeometric functions, Le Matematiche, 52(2) (1997), 297-310.

9. A.K. Rathie, K.S. Kumari and T.M. Vasudevan Nambisan, A study of I-functions of two variables, Le Matematiche 69(1) (2014), 285-305. 
10. D.R. Rhodes, On the spheroidal functions. J. Res. Nat. Bur. Standards. Sect. B 74 (1970), 187-209.

11. M. Saigo, M. and R.K. Saxena, Unified fractional integral formulas forthe multivariable H-function. J. Fractional Calculus 15 (1999), 91-107.

12. H.M. Srivastava, A multilinear generating function for the Konhauser set of biorthogonal polynomials suggested by Laguerre polynomial, Pacific. J. Math. 177(1985), 183-191.

13. H.M. Srivastava and M. Garg, Some integrals involving general class of polynomials and the multivariable Hfunction. Rev. Roumaine. Phys. 32 (1987), 685-692.

14. H.M. Srivastava and M.A.Hussain, Fractional integration of the H-function of several variables. Comput. Math. Appl. 30 (9) (1995), 73-85.

15. H.M. Srivastava and P.W. Karlsson, Multiple Gaussian Hypergeometric series. Ellis. Horwood. Limited. New-York, Chichester. Brisbane. Toronto, 1985.

16. H.M. Srivastava and R. Panda. Some expansion theorems and generating relations for the H-function of several complex variables. Comment. Math. Univ. St. Paul. 24 (1975), 119-137.

17. H.M. Srivastava and R.Panda, Some bilateral generating functions for a class of generalized hypergeometric polynomials, J. Reine Angew. Math. (1976), 265-274.

18. J.A. Stratton and L.J. Chu, Elliptic and spheroidal wave function, J. Math. and Phys. 20 (1941), 259-309. 